\title{
Semiconducting polymer compounds as sensitive layer for bolometric applications
}

\author{
A. Nocke ${ }^{1}$, M. Schossig ${ }^{1}$, M. Wolf ${ }^{1}$, H. Budzier ${ }^{1}$, K.-F. Arndt ${ }^{2}$, G. Gerlach ${ }^{1}$ \\ ${ }^{1}$ Technische Universität Dresden, Solid-State Electronics Laboratory, Dresden, Germany \\ ${ }^{2}$ Technische Universität Dresden, Institute of Physical Chemistry and Electrochemistry, Dresden, \\ Germany
}

\begin{abstract}
Our work investigates the ability to integrate conductive polymer compounds as self-supporting sensitive layers in microbolometers. An appropriate technology is presented including a sacrificial layer technique and a dielectrophoretic alignment procedure of the semiconducting filler particles. The polymer matrix causes mechanical stability of the compound and can be technologically modified using standard polymer processes.

The aligned filler material forms an electrically conducting network in the polymer matrix with a temperature coefficient of resistance (TCR) that yield the bolometric effect. The particle alignment allows the usage of significantly reduced amount of filler in contrast to non-aligned polymer compounds, which is advantageous for the other process steps. Investigated fillers are the semiconducting materials Tellurium (Te) and Vanadium-dioxide $\left(\mathrm{VO}_{2}\right)$. The electrical properties of the sensitive compounds are discussed. In comparison to the Te-compounds, the $\mathrm{VO}_{2}$-compounds have improved noise characteristics and higher TCR-values leading to improved sensor performance.
\end{abstract}

\section{Introduction}

Microbolometer arrays are the main sensor type being utilized in thermographic cameras. They are uncooled thermal detectors using the thermoresistive (bolometric) effect. In recent times, there is a growing interest in using thermographic cameras in various industrial and consumer fields like automotive, medical, thermal insulation, and security applications. Therefore, large afford has been done to reduce the production cost of microbolometers. The sensitive material of conventional microbolometers consists of inorganic materials like vanadium oxide $\left(\mathrm{VO}_{\mathrm{x}}\right)$ or amorphous silicon (a-Si) [1, 2]. Those materials have a temperature coefficient of resistance (TCR) of about $-(2 \ldots 3) \% / K$. The sensitive layer of each pixel is constructed as a self-supporting structure because of thermal insulation requirements needed for an adequate sensitivity. The corresponding MEMS process is complex and causes high production costs of such sensors.

In this work, an alternative concept is presented that is based on electrically conductive polymer compounds. These compounds are of major interest in recent research activities. They combine the advantages of polymer materials (easy processing and cost-effective materials) with the possibility to tailor the overall characteristics of the compound in terms of electrical, mechanical, or chemical properties. Therefore, polymer compounds are currently under investigation for various sensor applications, like biomedical [3], tactile [4], or gas sensing [5].

An electrically conductive polymer compound consists of an insulating polymer matrix and conducting filler particles. The filler materials under investigation for the bolometric application are the semiconductors Tellurium ( $\mathrm{Te}$ ) and Vanadium-dioxide $\left(\mathrm{VO}_{2}\right)$. Their electrical properties are similar to that of conventional sensitive materials used in microbolometers and thus are well suited for this application. Tellurium has a band gap of $0.33 \mathrm{eV}$ causing a bulk material TCR-value of $-2.3 \% / \mathrm{K}$ at room temperature [6]. It can be chemically synthesized and grows in elongated form due to its crystallographic characteristics [7]. The band gap of the semiconducting phase of $\mathrm{VO}_{2}$ at room temperature is $0.65 \mathrm{eV}$ [8]. This leads to a TCR-value of $-4.2 \% / \mathrm{K}$ for bulk material at room temperature. Additionally, they can be easily synthesized $(\mathrm{Te})$ or purchased $\left(\mathrm{VO}_{2}\right)$ as crystalline particles with dimensions in the micrometer range. The particle size is limited due to the maximum layer thickness of the sensitive layer in microbolometers to approximately $2 \mu \mathrm{m}$ (low heat capacity). 
For a homogeneous filler distribution, percolation theory can be used to describe the relationship between volume fraction $v$ and conductivitiy $\sigma[9]$ :

$$
\sigma \propto\left(v-v_{c}\right)^{t} .
$$

Here $v_{c}$ is the percolation threshold (critical volume fraction) and $t$ is a constant whose values depends on the compound properties. The percolation threshold $v_{c}$ represents the volume fraction, when the first conducting network is established. For spherical filler particles $v_{c}$ ranges from (15...30) vol.\% depending on the assumptions used in the model [9]. With increasing aspect ratio of the filler particles $v_{c}$ decreases. Another important influence for the percolation threshold is given by the geometrical dimensions of the compound. If the smallest dimension of the percolative system is in the same range as the particle size, the probability to form a conductive network perpendicular to this dimension decreases significantly with decreasing layer thickness [10].

The sensitive layers of microbolometers have a maximum thickness of $2 \mu \mathrm{m}$ and a typical pixel width of some tens of micrometers. Therefore, an electrically conductive polymer compound can only be achieved in one of the following cases:

(i) The filler particles have a very large aspect ratio and at least one of its dimensions is very small compared to the layer thickness. Carbon nanotubes (CNTs) can be such a filler particle. Research on CNT-filled polymer compounds for bolometric application has been performed by Aliev [11] and Itkis et al. [12]. The given results in their works are TCR-values of $-(0.1 \ldots 0.5 \% / K)$ and responsivities up to $150 \mathrm{VM}$ at room temperature.

(ii) If filler particles with another shape shall be used, they have to be aligned to form a particle chain between the electrodes. This can be achieved by dielectrophoresis [13]. The effect is based on the polarizability of a material in an inhomogeneous electric field. An attracting force results in higher electrical field intensity if the permittivity of the particle is larger than the permittivity of the surrounding medium. In this work dielectrophoretic alignment is used to form conducting networks of Te-rods and $\mathrm{VO}_{2}$-particles within the sensitive microbolometer layer.

\section{Experimental}

\subsection{Materials}

Te-rods were synthesized using chemical reduction of Telluric acid $\left(\mathrm{H}_{6} \mathrm{TeO}_{6}\right)$ with Hydrazine $\left(\mathrm{N}_{2} \mathrm{H}_{4}\right)$ [7]. The chosen synthesis procedure leads to a clean surface of the particles, which is desired for good electrical contacts. Crystalline $\mathrm{VO}_{2}$-particles were purchased from Aldrich having a size distribution from $100 \mathrm{~nm}$ to $10 \mu \mathrm{m}$. The $\mathrm{VO}_{2}$-powder was first processed by a sedimentation technique to remove the largest particles. SEM images and corresponding XRD-measurements of the particles are shown in Figure 1.

The Te-rods have an average diameter of $250 \mathrm{~nm}$ and an average length of $5 \mu \mathrm{m}$. The $\mathrm{VO}_{2}$-particles under investigation have a size distribution of $(0.5 \ldots 2) \mu \mathrm{m}$. The XRD-measurements verify the monocrystalline nature of both the particles.

The polymer matrix used is the photo resist AZ 1514 (Allresist). The main solid component in this photo resist is a novolak that can be cross-linked by heating above $150{ }^{\circ} \mathrm{C}$ to a phenolic resin.
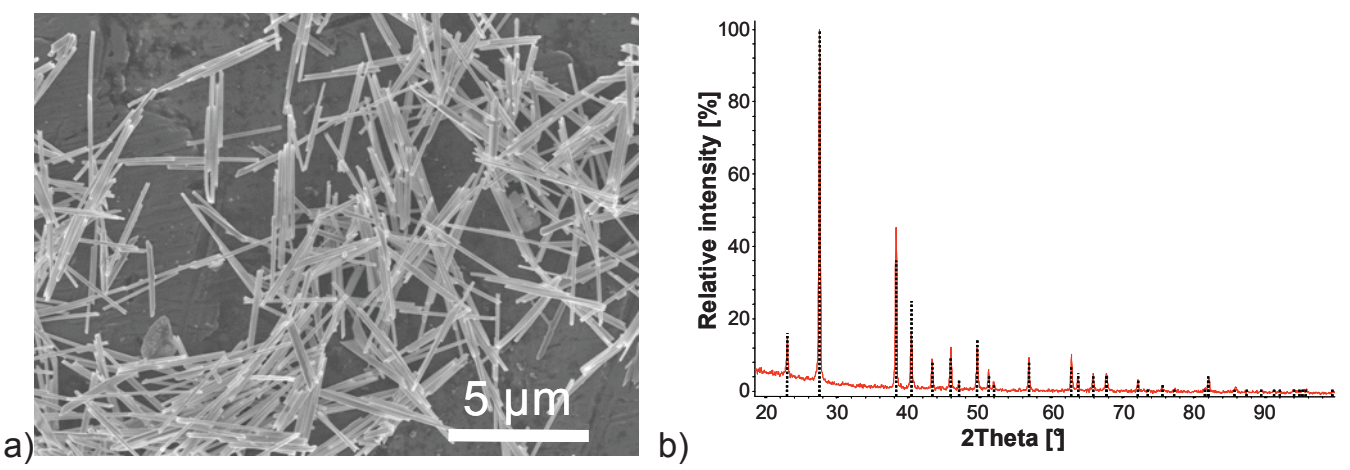

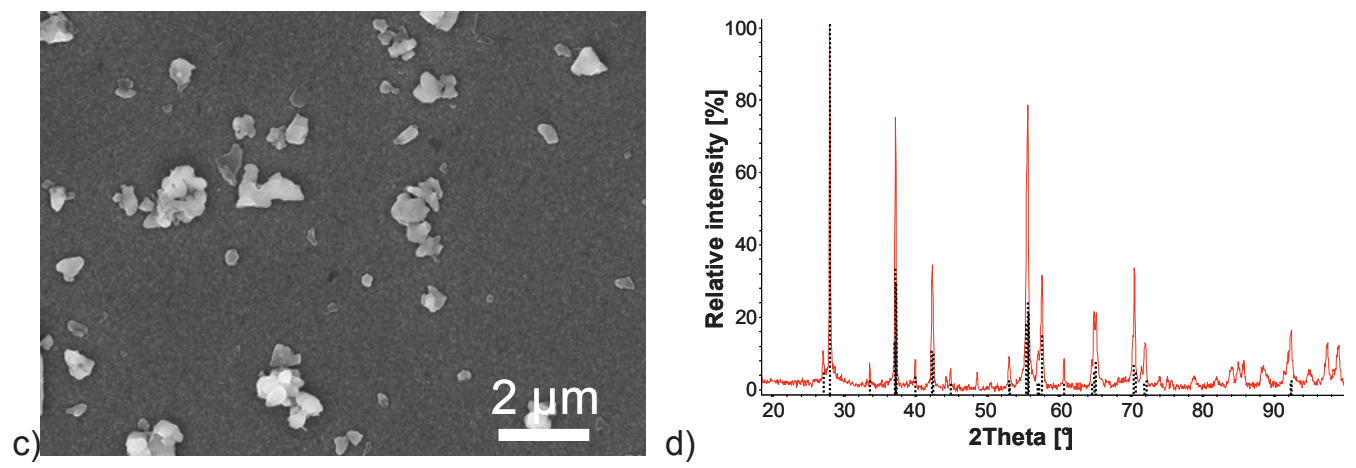

Figure 1: Scanning electron microscope images and results of XRD-measurements for $(a, b)$ Te-rods and $(\mathrm{c}, \mathrm{d}) \mathrm{VO}_{2}$-particles; Correlation of XRD-measurements (solid line) with theoretical peaks for mono-crystalline material (dotted line) are above $95 \%$.

\subsection{Technology}

Dielectrophoretic particle alignment of the particles is performed within the dissolved matrix polymer having a particle volume fraction of $0.1 \mathrm{vol} . \%$. To avoid electrolysis of the electrodes $(5 \mathrm{~nm} \mathrm{NiCr}$ as adhesion promoter and $5 \mathrm{~nm} \mathrm{Au}$ ) an $\mathrm{AC}$ voltage of $15 \mathrm{~V}$ with a frequency of $10 \mathrm{kHz}$ was applied during dielectrophoresis. A characteristic particle arrangement within the polymer compound is shown in Figure 2.
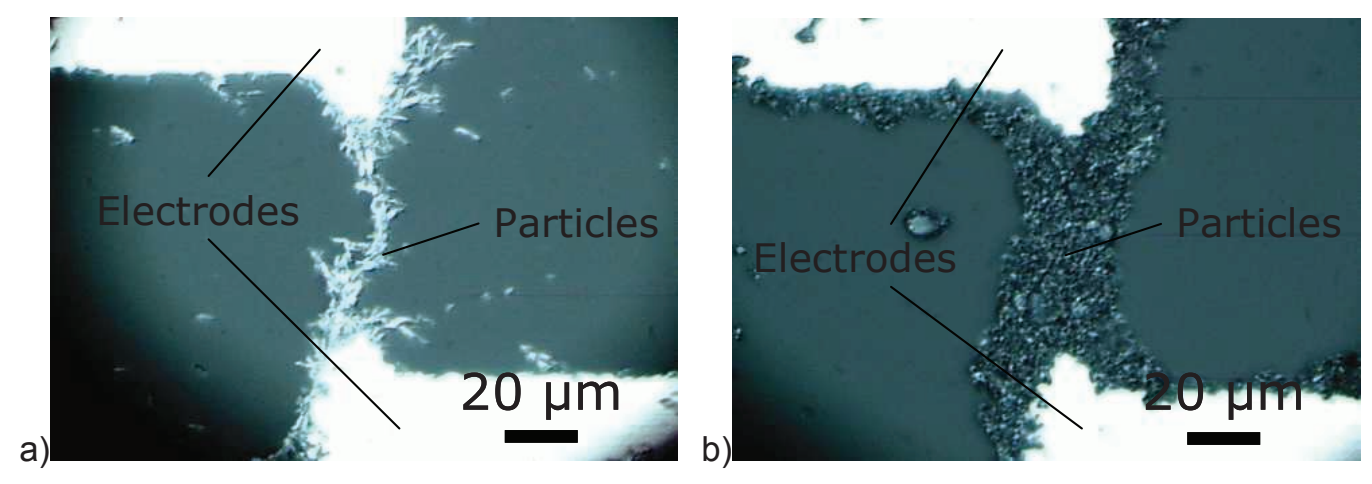

Figure 2: Optical images of polymer compounds with aligned (a) Te-rods and (b) $\mathrm{VO}_{2}$-particles.

To achieve the desired self-supporting sensitive structures a polymer-based technology is used. The scheme of this technology is shown in Figure 3. All process steps are applicable for a parallel production of multiple structures, which is essential for the processing of microbolometer arrays. A detailed explanation of the technological process is given in [14].
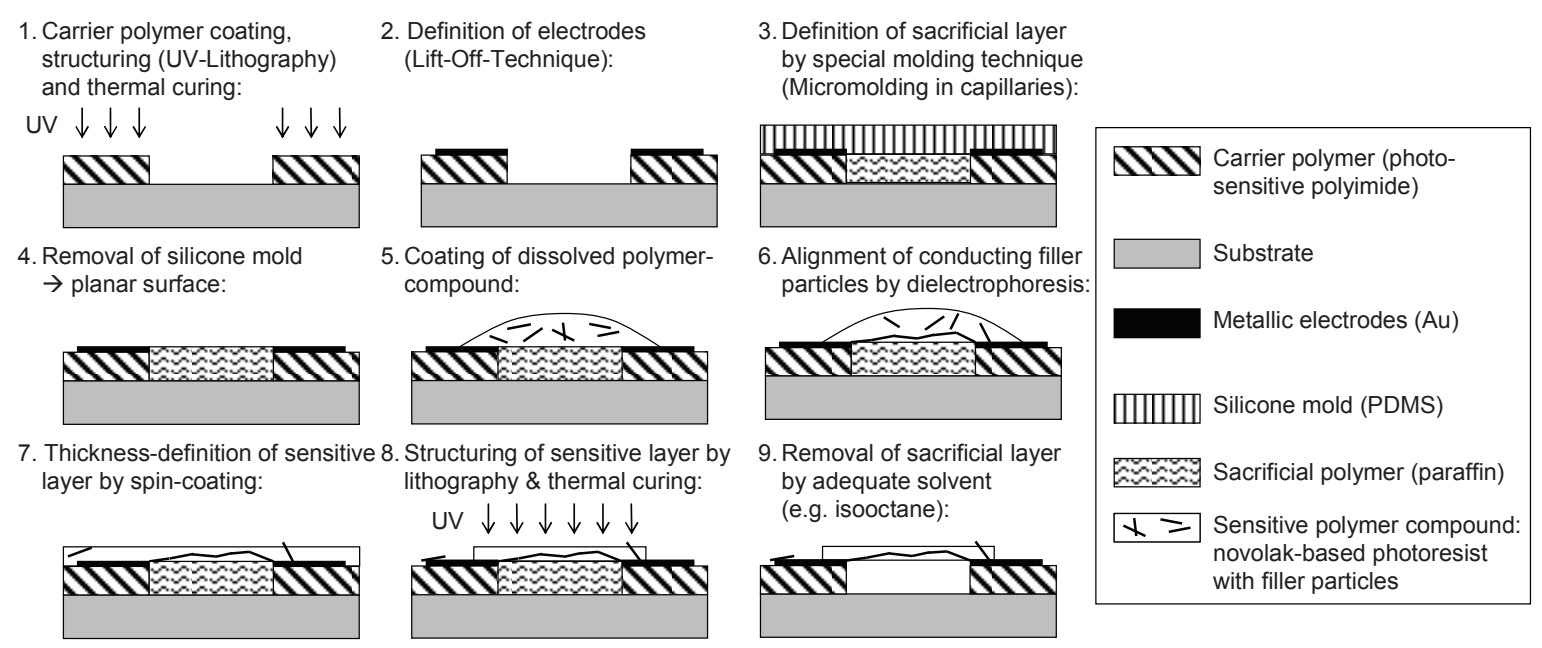

Figure 3: Technological process for a polymer-based microbolometer sensor element. 


\subsection{Characterization}

Electrical measurements were performed using the electrometer 617 with the testbox 8002A (Keithley) that is well suited for high ohmic samples. For temperature-dependant measurements a climate chamber $\mathrm{HCO020}$ (Voetsch) with additional humidity control was used. All measurements were performed at $0 \%$ relative humidity to minimize humidity influence. Noise characteristics were measured with the lock-inamplifier 7265 DSP (EG\&G Instruments).

\section{Results and discussion}

Microbolometer pixels are presented in Figure 4. Surface roughness of the supporting legs of the pixel is caused by diffraction during UV-lithography.

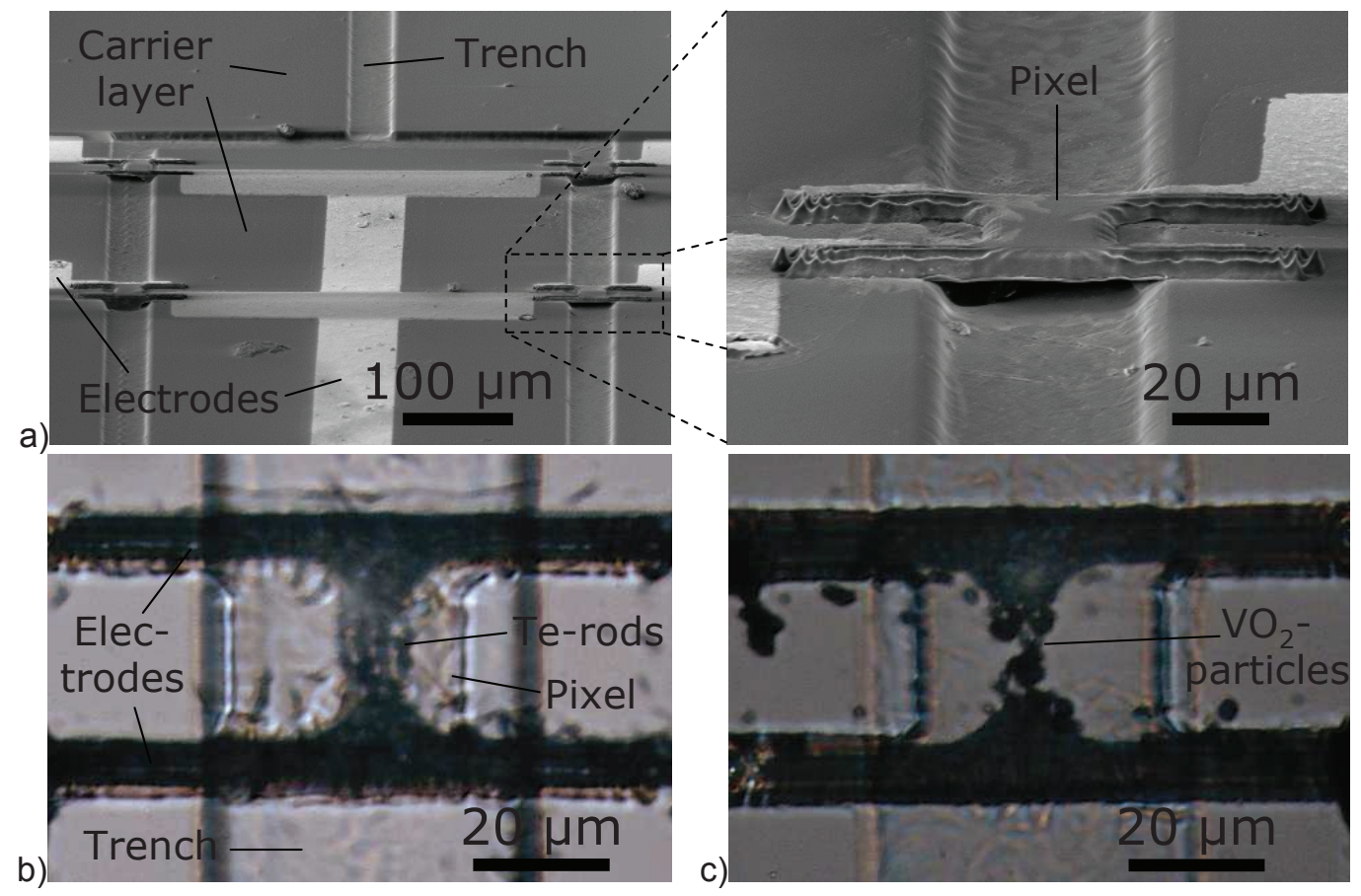

Figure 4: (a) Scanning electron microscope images of microbolometer array with $2 \times 2$ pixels $\left(\mathrm{VO}_{2}\right.$ particles) and optical images of microbolometer-pixels with aligned (b) Te-rods and (c) $\mathrm{VO}_{2^{-}}$ particles.

As can be seen in Figure 4, the contact electrodes are designed to be part of the self-supporting structure. The design is chosen to shorten the distance between the electrodes and thus increase the particle attraction during the dielectrophoretic alignment. Thermal properties of a self-supporting pixel are calculated in Table 1. The volume fraction of the filler particle in the sensitive area is unknown due to accumulation during the alignment process. However, its resulting volume fraction is assumed to be small and is therefore neglected in the calculations.

Table 1: Calculation of thermal parameters for a microbolometer pixel; Value of thermal conductivity for thin film gold taken from empirical model by Qiu [15] (bulk value: $317 \mathrm{~W} /(\mathrm{m} \mathrm{K})$ ).

\begin{tabular}{llll}
\hline Parameter & Matrix polymer & Electrode $(\mathrm{Au})$ & Layer stack \\
\hline Specific heat capacity $[\mathrm{J} /(\mathrm{kg} \mathrm{K})]$ & 1670 & 128 & \\
Thermal conductivity $[\mathrm{W} /(\mathrm{m} \mathrm{K})]$ & 0.20 & 48.3 & \\
Density $\left[\mathrm{g} / \mathrm{cm}^{3}\right]$ & 1.14 & 19.3 & \\
Length of supporting leg $[\mu \mathrm{m}]$ & 7.5 & 7.5 & \\
Width of supporting leg $[\mu \mathrm{m}]$ & 7.5 & 7.5 & \\
Layer thickness $[\mu \mathrm{m}]$ & 2 & 0.005 & $4.6^{*} 10^{-6}$ \\
Pixel size $\left[\mu \mathrm{m}^{2}\right]$ & $40^{*} 40$ & $40^{*} 40$ & $6.1^{*} 10^{-9}$ \\
Thermal conductance $[\mathrm{W} / \mathrm{K}]$ & $2.7^{*} 10^{-6}$ & $1.9^{*} 10^{-6}$ & $7.4^{*} 10^{-12}$ \\
Heat capacity $C_{t h}[\mathrm{~J} / \mathrm{K}]$ & $6.1^{*} 10^{-9}$ &
\end{tabular}


Temperature dependant current-voltage characteristics and noise spectra for the polymer compounds with aligned filler particles are shown in Figure 5.

a)

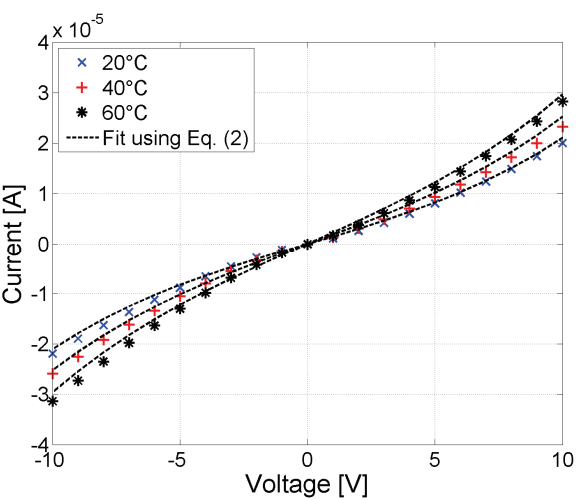

c)

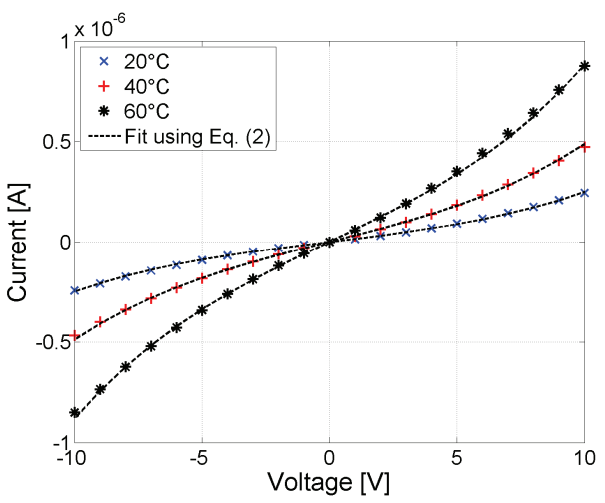

b)
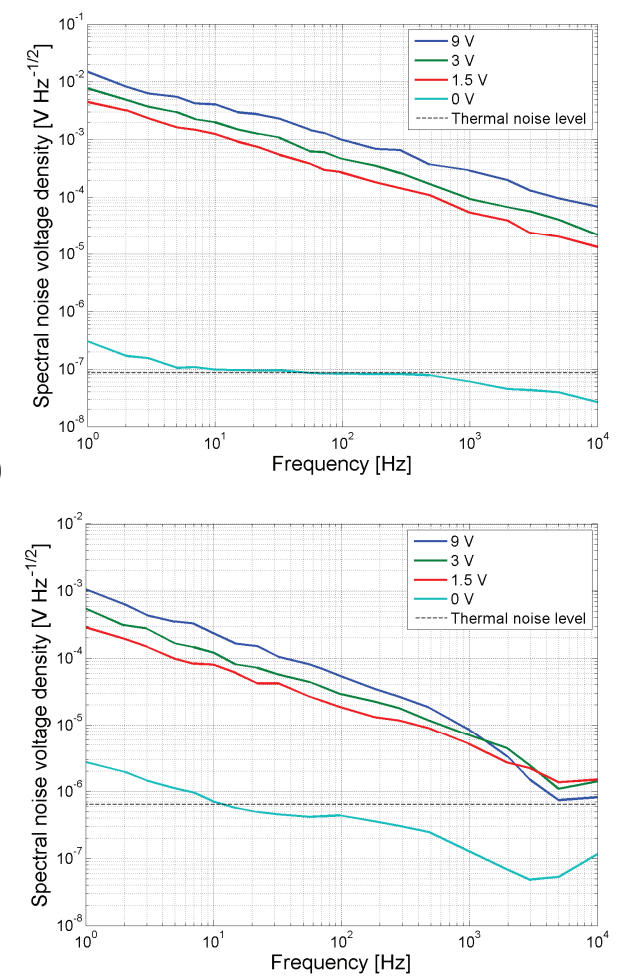

Figure 5: Temperature dependant current-voltage characteristics and noise spectra for polymer compounds with aligned $(a, b)$ Te-rods and (c,d) $\mathrm{VO}_{2}$-particles.

The conducting mechanism in an electrically conductive polymer compound is mainly determined by the intrinsic properties of the filler particles and the hopping conduction between neighbouring particles. Considering semiconducting particles, both conduction mechanisms are thermally activated and have a characteristic energy barrier: the band gap $E_{g}$ in semiconductors and the hopping activation energy $W$ in disordered materials. Hopping conduction $\sigma_{H}$ at room temperature can be expressed as [16]

$$
\sigma_{H} \propto \exp \left(\frac{-W}{k T}\right) \sinh \left(\frac{e R E}{k T}\right),
$$

where $k$ is the Boltzmann constant, $T$ is the temperature, $e$ is the elementary charge, $R$ is the average hopping distance, and $E$ is the applied electric field. The intrinsic conductivity of semiconductors can be derived from this equation by considering only the first exponential term and substituting $W$ with $E_{g} / 2$. Therefore, both influences cannot be distinguished. As can be seen in Figure 5, equation (2) is well suited to describe the overall temperature dependant current-voltage characteristics of the investigated compounds. TCR-values of the compounds with aligned Te-rods and $\mathrm{VO}_{2}$-particles are $-1.4 \% / \mathrm{K}$ and $-3.9 \% / \mathrm{K}$, respectively. The similarity of the TCR-values for the aligned $\mathrm{VO}_{2}$-compounds and the bulk material $(-4.2 \% / \mathrm{K})$ indicates that conduction is mainly determined by the intrinsic semiconducting particles. The corresponding difference for the Te-rods is much higher (bulk: $-2.3 \% / \mathrm{K}$ ). Therefore, hopping transitions above energy barriers dominate the conduction mechanism in this compound. High energy barriers in this compound can be caused by the high anisotropy of the Te-rods leading to small contact areas or a small oxidation layer on the surface.

Considering noise characteristics the same explanations are valid to describe the higher noise level of the Te-compound in contrast to the $\mathrm{VO}_{2}$-compound (with bias voltage). Both polymer compounds show the characteristic 1/f-noise behaviour being typical in disordered media [17].

Sensor properties are summarized in Table 2. Responsivity and detectivity of the presented microbolometer pixels are calculated using the values from Table 1 and the electric measurements from Figure 5. 
Table 2: Measured and calculated parameters for the microbolometer pixels.

\begin{tabular}{lll}
\hline Parameter & Te-rods & VO $_{2}$-particle \\
\hline Bolometer resistance $(293 \mathrm{~K})[\Omega]$ & $7.5 \pm 1.6^{*} 10^{6}$ & $28 \pm 10^{*} 10^{7}$ \\
TCR $(293 \mathrm{~K})[\% / \mathrm{K}]$ & $-1.4 \pm 0.1$ & $-3.9 \pm 0.1$ \\
Bias voltage $[\mathrm{V}]$ & 9 & 9 \\
Thermal conductance $[\mathrm{W} / \mathrm{K}]$ & $4.6^{*} 10^{-6}$ & $4.6^{*} 10^{-6}$ \\
Heat capacity $[\mathrm{J} / \mathrm{K}]$ & $6.1^{*} 10^{-9}$ & $6.1^{*} 10^{-9}$ \\
Thermal time constant $[\mathrm{s}]$ & $1.3^{*} 10^{-3}$ & $1.3^{*} 10^{-3}$ \\
Responsivity $(f=50 \mathrm{~Hz})[\mathrm{V} / \mathrm{W}]$ & $2.5^{*} 10^{4}$ & $7.6^{*} 10^{4}$ \\
Detectivity $(f=50 \mathrm{~Hz})\left[\mathrm{cm} \mathrm{Hz}{ }^{1 / 2} / \mathrm{W}\right]$ & $5.9^{*} 10^{4}$ & $3.5^{*} 10^{6}$ \\
\hline
\end{tabular}

\section{Summary}

Polymer based microbolometer arrays with an electrically conductive polymer compound as sensitive layer are presented in this work. Major focus is on the electrical properties of the aligned particles in the polymer compound. Polymer compounds with aligned $\mathrm{VO}_{2}$-particles have a higher TCR-value and lower noise characteristics in contrast to compounds with aligned Te-rods and therefore improved sensor characteristics. The responsivity of microbolometers with $\mathrm{VO}_{2}$-compounds is $7.6^{*} 10^{4} \mathrm{~V} / \mathrm{W}$. Compared to CNT-filled polymer compounds this value is much higher. Maximum detectivities of $3.5^{*} 10^{6} \mathrm{~cm} \mathrm{~Hz} / 1 / 2 \mathrm{~W}$ are calculated. In contrast to conventional microbolometers, this value is comparatively low mainly caused by high noise levels of the polymer compounds.

\section{Acknowledgments}

The authors gratefully acknowledge financial support by the Saxon State Ministry of Science and Art and from German Research Foundation (DFG).

\section{References:}

1. $\quad$ Ambrosio, R. et al.: physica status solidi (c) 7 (2010) 3-4, pp. 1180-1183.

2. Niklaus, F., Vieider, C. and Jakobsen, H. In: Proceedings of SPIE 6836. 2007. Beijing, China, pp. 68360D-15.

3. Nambiar, S. and Yeow, J.T.W.: Biosensors and Bioelectronics 26 (2011) 5, pp. 1825-1832.

4. Cheng, M.Y. et al.: Sensors and Actuators A: Physical, In Press, Corrected Proof.

5. $\quad$ Zhang, T. et al.: Nanotechnology 18 (2007) 16, pp. 165504-6.

6. $\quad$ Nussbaum, A.: Physical Review 94 (1954) 2, pp. 337-342.

7. Mayers, B. and Xia, Y.N.: Journal of Materials Chemistry 12 (2002) 6, pp. 1875-1881.

8. Berglund, C.N. and Guggenheim, H.J.: Physical Review 1 (1969) 3, pp. 1022-1033.

9. Sahimi, M.: Applications of Percolation Theory. London: Taylor \& Francis 1994.

10. Butera, A., Klemmer, T.J. and Barnard, J.A.: Journal of Applied Physics 83 (1998) 9, pp. 48554861.

11. Aliev, A.E.: Infrared Physics \& Technology 51 (2008) 6, pp. 541-545.

12. Itkis, M.E. et al.: Science 312 (2006) 5772, pp. 413-416.

13. Jones, T.B.: Electromechanics of Particles. Cambridge, New York: Cambridge University Press 1995.

14. Nocke, A. et al. In: Proceedings of SPIE 7481. 2009. Berlin, Germany, pp. 74810I-9.

15. Chen, G. and Hui, P.: Applied Physics Letters 74 (1999) 20, pp. 2942-2944.

16. Mott, N.F. and Davis, E.A.: Electronic Processes in Non-Crystalline Materials. 2nd ed. Oxford: Clarendon Press 1979.

17. Dziedzic, A. and Kolek, A.: Journal of Physics D-Applied Physics 31 (1998) 17, pp. 2091-2097. 